\title{
P-0903 - The association between diabetes and disability: what is the contribution of diabetes risk factors and complications?
}

Maryam Tabesh ${ }^{1,2}$,Jonathan E. Shaw ${ }^{1,2}$, Paul Z. Zimmet ${ }^{1}$, Stefan Söderberg ${ }^{1}$, Digsu N. Koye ${ }^{1,2}$, Sudhir Kowlessur ${ }^{3}$,

Noorjehan Joonas ${ }^{3}$, Ameena Sorefan ${ }^{3}$, Praneel Gayan ${ }^{3}$, George M.M. Alberti ${ }^{4}$, Jaakko Tuomilehto ${ }^{5}$, Dianna J. Magliano ${ }^{1,2}$

${ }^{1}$ Baker Heart and Diabetes Institute, Melbourne, Australia, ${ }^{2}$ Department of Epidemiology and Preventive Medicine, Monash University, Melbourne, Australia, ${ }^{3}$ Ministry of Health and Quality of Life, Republic of Mauritius, ${ }^{4}$ Department of Endocrinology and Metabolism, St Mary's Hospital and Imperial College, London, UK, ${ }^{5}$ Chronic Disease Prevention Unit, National Institute for Health and Welfare, Helsinki, Finland

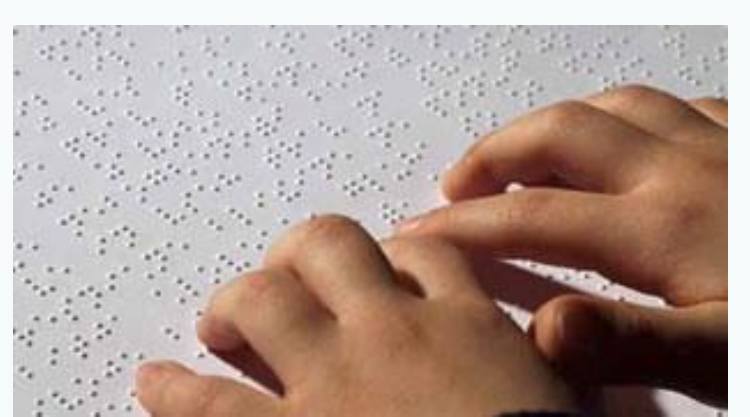

Background:

People with diabetes have two to three fold higher risk of physical disability. Diabetes is the leading cause of disabling diseases such as cardiovascular disease, renal failure, blindness and lower limb amputation. Understanding the factors that explain the association between diabetes and disability might provide insight into strategies to reduce the burden of disability and its related cost and improving quality of life among people with diabetes.

Mauritius is a multi-ethnic nation with a population of 1.3 million people comprising diverse ethnicities including South Asians (Indian origin), Creoles of mainly African origin and Chinese. The age- and sex standardized prevalence of Type 2 diabetes in the Mauritian population aged 18-74 years was estimated at $19.2 \%$, which makes this one the highest in terms of diabetes prevalence in the world (1). The high prevalence of diabetes and concomitant diseases together with ethnic diversity in Mauritius provides an ideal setting to investigate the association of disability with diabetes in a middle-income country.

Methods:

A national population-based survey was conducted in Mauritius in 2015. The assessments included a blood sample, anthropometric and blood pressure measurements, and interviewer-administered questionnaires. Disability was estimated in adults aged $\geq 50$ years using the Katz index of independence in activities of daily living (Katz ADL) questionnaire. This instrument rates adequacy of performance in six major activities of daily living including: walking, bathing, transferring, toileting, dressing, and eating.

A logistic regression model was used to examine the association between diabetes and disability, with disability as the main outcome of interest. Further, a series of logistic regression models were conducted and adjusted for the different variables. To quantify the contribution of risk factors to the association of disability with diabetes, the percentage of excess odds of disability related to diabetes in each model were calculated and compared to the base model

Table 1: Odds ratios (95\% Cis) for the association of diabetes

Total population

OR

Model 1: Adjusted for age, sex, education and smoking

$1.67(1.34,2.08)$

$1.43(1.31,1.80)$

$1.52(1.20,1.93)$

$1.46(1.15,1.85)$

0.001

$1.29(1.01,1.67)$

0.045

Model 1+ chronic disease

$1.29(1.01,1.67)$ 0.045

Table 2: The percentage of excess odds accounted for by risk factor adjustment $(95 \% \mathrm{CI})$

\begin{tabular}{ll} 
Model 1: Adjusted for age, sex, education and smoking & Total population \\
\hline
\end{tabular}

Model $1+$ BMI and waist

Model 1+ blood pressure and lipids

Model 1+ chronic disease*

Adjusted for all variables

\begin{abstract}
$29.8(17.9,41.8)$
\end{abstract}
$17.7(4.4,39.3)$

$25.6(6.4,38.1)$

48.9 (23.6, 98.2)
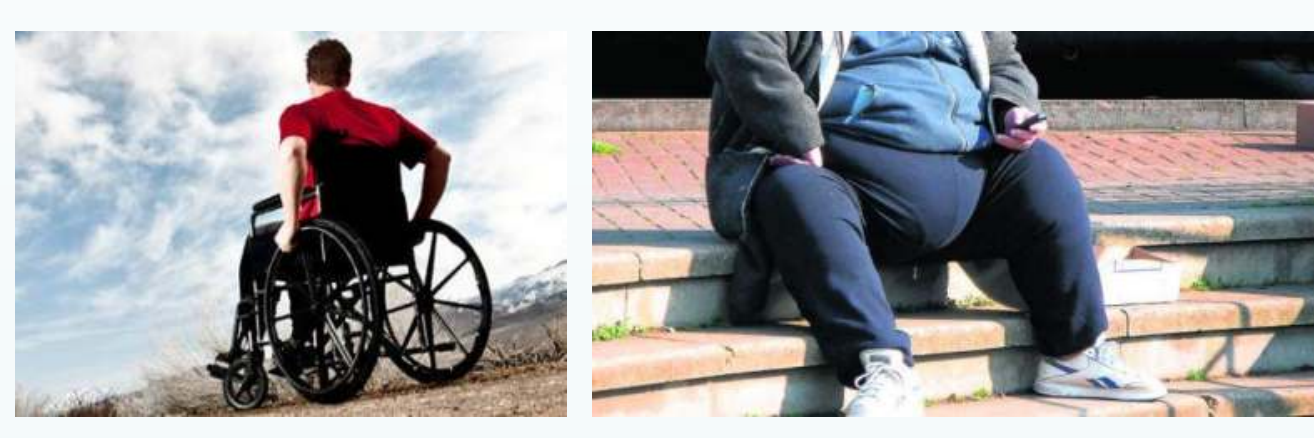

Results: There was a significantly higher prevalence of disability among women $6.4 \%(95 \%$ CI 14.8, 18.0)] than men $[9.1 \%(95 \%$ CI $7.8,10.6)]$.

of diabetes with disability was stronger among African (95\% CI 1.18, 1.95)]. Asability with diabetes among South Asians and resulted in a nonsificant association [OR: 1.21 (95\% CI 0.91- 1.62)]. In contrast, in frican Creoles, diabetes remained significantly

BMI and waist circumference together explained nearly $33 \%$ of the sociation between diabetes and disability.

*chronic disease: history of CVD, asthma, GFR and depression

1) Tabesh M, Shaw J.E, Zimmet P.Z, Soderberg S, Kowlessur S, Timol M, Joonas N, Alberti G.M.M, Tuomilehto J, Shaw B.J, Magliano D.J, Meeting American Diabetes Association diabetes management targets: trends in Mauritius, Diebet Med. 2017 Aug 9.

\begin{tabular}{|ll}
\hline Africans & \\
\hline OR & $P$ \\
$2.61(1.54,4.43)$ & $<0.001$ \\
$2.17(1.24,3.79)$ & 0.006 \\
$2.54(1.44,4.51)$ & 0.001 \\
$2.28(1.29,4.03)$ & 0.005 \\
$2.06(1.08,3.91)$ & 0.027
\end{tabular}

\section{South Asians}

Base model

33.3 (18.0, 53.4)

$22.9(0.0,52.3)$

26.6 (10.2, 42.9)

$53.7(21.2,100)$
Africans

Base model

19.1 (3.6, 44.7)

$2.7(0.0,36.3)$

$14.2(0.0,38.4)$

$24.7(0.0,71.2)$ 